01,09

\title{
Электронная структура и оптические спектры соединений GdFeAl и GdFeSi
}

\author{
() Ю.В. Князев ${ }^{1}$, А.В. Лукоянов ${ }^{1,2}$, Ю.И. Кузьмин ${ }^{1}$, А.Г. Кучин ${ }^{1}$, С.П. Платонов ${ }^{1}$ \\ ${ }^{1}$ Институт фризики металлов им. М.Н. Михеева УрО РАН, \\ Екатеринбург, Россия \\ ${ }^{2}$ Уральский федееральный университет им. Б.Н. Ельцина, \\ Екатеринбург, Россия \\ E-mail: knyazev@imp.uran.ru
}

Поступила в Редакцию 5 февраля 2021 г.

В окончательной редакции 5 февраля 2021 г.

Принята к публикации 11 февраля 2021 г.

Представлены результаты исследований электронной структуры и оптических свойств интерметаллических соединений $\mathrm{GdFeAl}$ и GdFeSi. В рамках метода DFT $+U$ с учетом влияния сильных электронных взаимодействий в $4 f$-оболочке $\mathrm{Gd}$ рассчитаны спин-поляризованные плотности электронных состояний и спектры межзонной оптической проводимости. Методом эллипсометрии в интервале длин волн $0.22-16 \mu \mathrm{m}$ изучены оптические свойства данных материалов. На основе сравнительного анализа экспериментальных и теоретических спектров обсуждается природа квантового поглощения света. Показано, что рассчитанные плотности электронных состояний позволяют качественно интерпретировать основные особенности частотных зависимостей оптической проводимости.

Ключевые слова: соединения $\mathrm{GdFeAl}$ и $\mathrm{GdFeSi}$, оптические свойства, электронная структура.

DOI: 10.21883/FTT.2021.06.50925.019

\section{1. Введение}

Изучение физических свойств семейства тройных интерметаллидов $R T X(R-$ редкоземельный металл, $T$ и $X$ соответственно, $d$ - и $p$-металлы) вызывает значительный интерес благодаря многообразию их магнитных и электронных характеристик, а также возможностям для практического использования (см. обзоры [1-3]). В последние годы было синтезировано и исследовано большое количество таких соединений, показано, что уникальность их свойств зависит от особенностей гибридизации и взаимодействия локализованных $4 f$-электронов редкоземельного металла с электронами переходных металлов. К материалам подобного типа относятся ферромагнитные интерметаллиды GdFeAl и $\mathrm{GdFeSi}$ (точки Кюри $T_{C}$ соответственно, 265 и $130 \mathrm{~K}$ ). В данных соединениях наблюдается целый ряд физических явлений, связанных с образованием различных кристаллических структур и магнитных фаз [4-9]. При этом магнитные моменты обеих систем определяются атомами $\mathrm{Gd}$, поскольку атомы $\mathrm{Fe}$, вследствие полного заполнения $3 d$-зоны, не обладают магнитным моментом. Необходимо особо отметить такие свойства, как аномально большие значения магнетокалорических эффектов вблизи точек магнитного упорядочения [10-15] и высокую способность к абсорбции атомарного водорода $[16-18]$, позволяющие рассматривать эти сплавы в качестве функциональных материалов. Соединение $\mathrm{GdFeAl}$ в зависимости от условий приготовления может кристаллизоваться в кубической структуре типа $\mathrm{MgCu}_{2}$ или в гексагональной $\mathrm{MgZn}_{2}$ структуре. Возможно так- же совместное существование обеих кристаллических фаз. Интерметаллид $\mathrm{GdFeSi}$, в свою очередь, упорядочивается в тетрагональной структуре типа CeFeSi. Сплавление данных соединений с другими металлами, a также внешние воздействия приводят к заметным изменениям кристаллических параметров и магнитных характеристик [5,14,16-20]. Например, замещение $\mathrm{Fe}$ атомами $\mathrm{Mn}$ в GdFeSi влечет за собой увеличение размеров решетки, рост результирующего магнитного момента и значительное повышение $T_{\mathrm{C}}[7,19]$. При абсорбции водорода в данном соединении происходит его структурный переход к другой тетрагональной фазе типа $\mathrm{ZrCuSiAs} \mathrm{c} \mathrm{анизотропным} \mathrm{изменением} \mathrm{параметров}$ решетки и резким уменьшением температуры Кюри, а механическое измельчение приводит к образованию фазы аморфного магнетизма с $T_{\mathrm{C}}=65 \mathrm{~K}[17,18]$.

Большая вариативность физических свойств этих материалов стимулирует интерес к изучению их электронной структуры. Особенности распределения $\mathrm{Fe} 3 d$ и $\mathrm{Gd} 4 f$-состояний в валентной зоне энергетического спектра GdFeAl были исследованы методом рентгеновской фотоэмиссионной спектроскопии [21]. Теоретические расчеты зонной структуры $\mathrm{GdFeSi}$, показавшие существенное сходство, были выполнены для объемного [22,23] и наноструктурного [24] состояний материала. В настоящей работе для изучения электронных характеристик данных интерметаллидов используется метод, совмещающий первопринципные спин-поляризованные расчеты плотностей электронных состояний и экспериментальные исследования оптических свойств. 
Цель исследования - определить степень корреляции теоретической модели расчета, учитывающей сильные электронные взаимодействия в $4 f$-оболочке $\mathrm{Gd}$, с наблюдаемыми спектрами межзонного оптического поглощения.

\section{2. Образцы и методы}

Поликристаллические образцы соединений $\mathrm{GdFeAl}$ и $\mathrm{GdFeSi}$ были приготовлены из высокочистых металлов методом дуговой плавки в атмосфере чистого аргона. Полученные сплавы подвергались гомогенизирующему отжигу в течение восьми суток при температуре $\sim 1100 \mathrm{~K}$ с последующей закалкой в воде. Для определения фазового состава, типа кристаллической структуры и параметров кристаллической решетки применялся рентгеноструктурный анализ с использованием диффрактометра PANanalitical Empyrean при $\mathrm{CuK} \alpha$-излучении. Расчет параметров решетки и анализ фазового состава сплавов осуществлялся на базе программ HighScore v.4.x. Результаты исследования показали, что сплав GdFeAl кристаллизуется в двухфазном состоянии: помимо гексагональной $\operatorname{MgZn}_{2}(P 63 / m m c)$ фазы (массовая доля 55.9\%) реализуется также кубическая структура типа $\mathrm{MnCu}_{2}(F d 3 m)(44.1 \%)$. В свою очередь, соединение GdFeSi формируется в тетрагональной кристаллической структуре типа $\mathrm{CeFeSi}(P 4 / n m m)$ с $8 \%$ примесью гексагональной фазы $\mathrm{Gd}_{5} \mathrm{Si}_{3}$ со структурой типа $\mathrm{Mn}_{5} \mathrm{Si}_{3}\left(P 6_{3} / \mathrm{mcm}\right)$. Полученные параметры кристаллических решеток были использованы при расчете электронной структуры соединений.

Электронная структура тройных интерметаллидов $\mathrm{GdFeAl}$ и GdFeSi была рассчитана при помощи пакета программ QUANTUM ESPRESSO [25] в рамках метода DFT $+U[26]$ на базе приближения обобщенной градиентной поправки версии Perdew-Burke-Ernzerhof (PBE) [27]. В расчетах были применены псевдопотенциалы из стандартной библиотеки QUANTUM ESPRESSO и пакета [28] на основе метода присоединенных плоских волн (PAW). Для процедуры интегрирования в обратном пространстве была задана сетка из $8 \times 8 \times 8 k$-точек. Для $4 f$-оболочки Gd электронные корреляции в методе $\mathrm{DFT}+U$ учитывались при выборе параметров кулоновского $6.7 \mathrm{eV}$ и обменного $0.7 \mathrm{eV}$ взаимодействий.

Оптические свойства образцов изучены при комнатной температуре в интервале длин волн $0.22-16 \mu \mathrm{m}$ (энергия фотонов $E=0.078-5.64 \mathrm{eV}$ ). В эксперименте использовался эллипсометрический метод с вращающимся анализатором, основанным на определении разности фаз и амплитуды световых волн $s$ - и $p$-поляризаций, отраженных от зеркальной плоскости образца [29]. Указанные величины, зависящие от частоты света $\omega$, позволяют рассчитать оптические постоянные исследуемых соединений - показатели преломления $n(\omega)$ и $k(\omega)$. По этим характеристикам вычислены дисперсионные зависимости оптической проводимо- сти $\sigma(\omega)=n k \omega / 2 \pi$, параметра, наиболее выразительно описывающего частотные и амплитудные особенности спектрального отклика отражающей среды. В отличие от статической проводимости, оптическая проводимость зависит не только от плотности электронных состояний на уровне Ферми $E_{\mathrm{F}}$, но и от характера ее распределения во всем исследуемом диапазоне энергий световых волн.

\section{3. Обсуждение результатов}

Полные $N(E)$ и парциальные для $\mathrm{Gd} 4 f, 5 d, 6 p, 6 s$, $\mathrm{Fe} 3 d, 4 p, 4 s$ и $\mathrm{Al}(\mathrm{Si}) 3 s, 3 p$ плотности электронных состояний ферромагнитных соединений GdFeAl и GdFeSi, рассчитанные для доминирующих кристаллических фаз, приведены на рис. 1-3. Они представляют собой совокупности двух систем энергетических полос со спинами, условно ориентированными вдоль $(\uparrow)$ и против $(\downarrow)$ направления спонтанной намагниченности. При этом основной вклад в полную плотность состояний формируется за счет $\mathrm{Gd} 4 f, 5 d$ и $\mathrm{Fe} 3 d$-электронов. Обращает внимание близкое сходство зависимостей $N(E)$ для кубической и гексагональной фаз $\mathrm{GdFeAl}$, проявляемое в обоих спиновых направлениях. При этом структуры $N \uparrow(E)$ и $N \downarrow(E)$ в данных фазах кардинально различа-

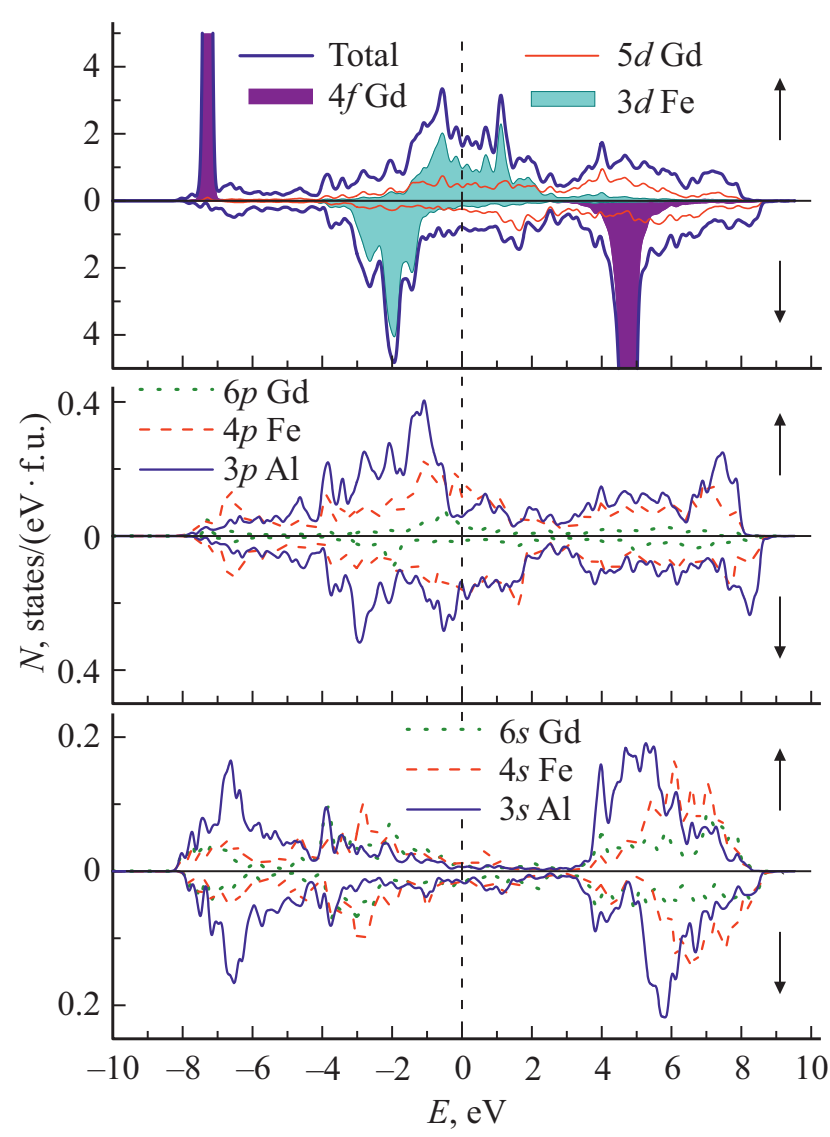

Рис. 1. Полные и парциальные плотности электронных состояний соединения $\mathrm{GdFeAl}$ (кубическая фаза типа $\mathrm{MgCu}_{2}$ ). 


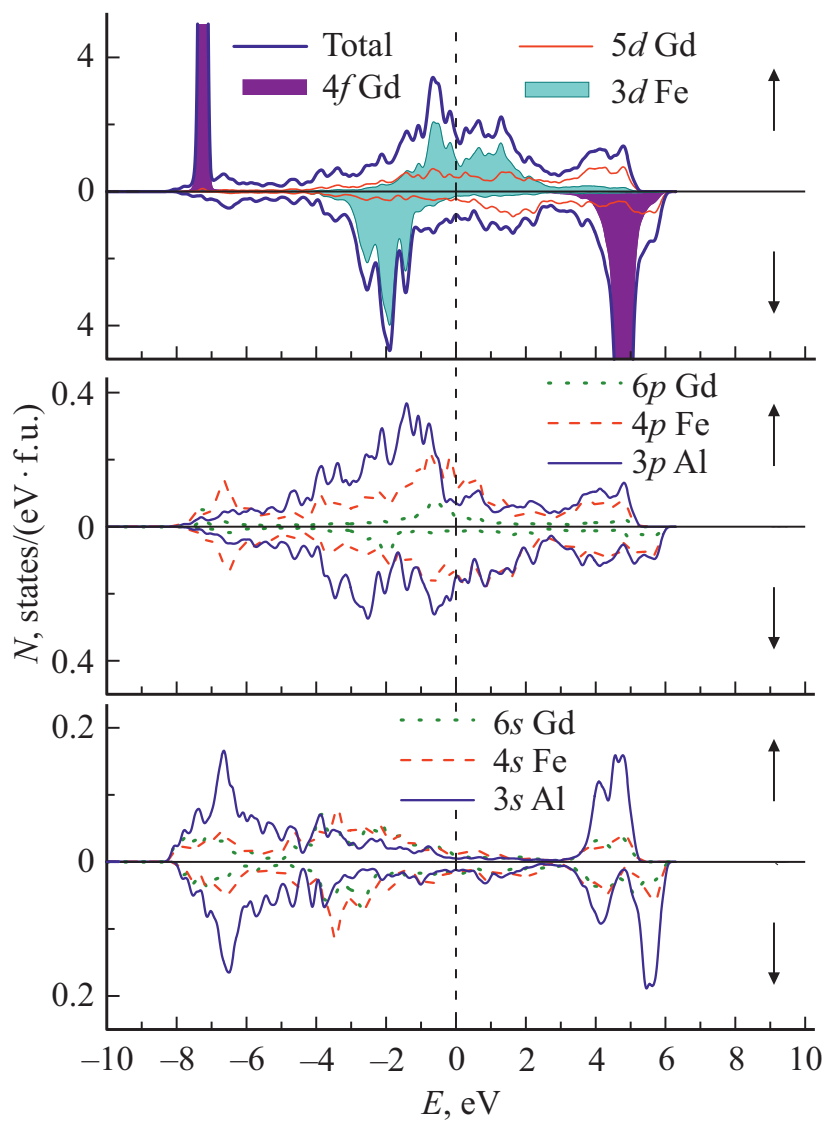

Рис. 2. Полные и парциальные плотности электронных состояний соединения GdFeAl (гексагональная фаза типа $\mathrm{MnZn}_{2}$ ).

ются из-за сильной спиновой поляризации ионов железа. Если протяженные участки $\mathrm{Fe} 3 d \uparrow$-зоны с повышенной интенсивностью расположены по обе стороны от $E_{\mathrm{F}}$, то Fe $3 d \downarrow$-зоны почти полностью заполнены. Уровень Ферми в этой системе энергетических полос проходит выше верхней границы данных электронных состояний. Узкие интенсивные максимумы, связанные с $4 f$-состояниями $\mathrm{Gd}$, расположены в зависимостях $N(E)$ обеих структурных фаз при одинаковых энергиях: в $\uparrow$-системе зон в интервале $7-8 \mathrm{eV}$ ниже $E_{\mathrm{F}}$, а в $\downarrow$-системе - при $4.8 \mathrm{eV}$ выше $E_{\mathrm{F}}$. Энергетический интервал разделяющий данные состояния, соответствует величине $U$, используемой в расчете. Отметим, что полученное в расчете значение энергии локализации заполненных $4 f$-состояний коррелирует с результатами рентгеновской фотоэмиссии [21], где экспериментально оцениваемая величина энергии связи данных электронов близка к $8 \mathrm{eV}$. Структура зависимостей $N(E)$, рассчитанных для тетрагонального $\mathrm{GdFeSi}$, существенно отличается. В данном соединении спектры $N \uparrow(E)$ и $N \downarrow(E)$, формируемые преимущественно $\mathrm{Gd} 5 d$ и $\mathrm{Fe} 3 d$-зонами (рис. 3 ), близки по форме, а уровень Ферми в обоих спиновых направлениях расположен на расстоянии $\sim 0.5 \mathrm{eV}$ от максимальных значений плотности состояний. Интенсивные пики, соответствующие $4 f \uparrow$ - и $4 f \downarrow$-состояниям, локализованы в $N(E)$ данного соединения при тех же энергиях, что и в $\mathrm{GdFeAl}$. Зоны, формируемые $\mathrm{Gd} 6 p, 6 s$, Fe $4 p, 4 s$, а также $\mathrm{Al}(\mathrm{Si}) 3 p, 3 s$-состояниями, представляют протяженные полосы слабой интенсивности и дают малый вклад полную плотность состояний.

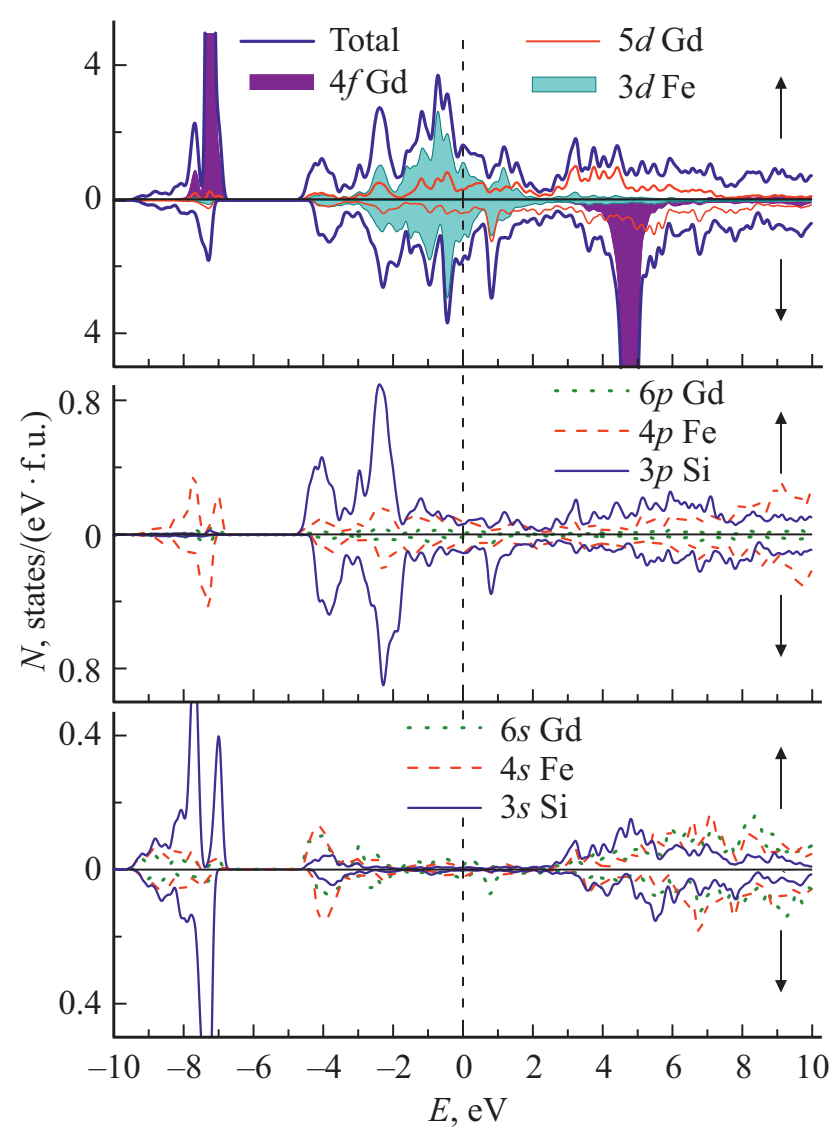

Рис. 3. Полные и парциальные плотности электронных состояний соединения GdFeSi (тетрагональная фаза типа $\mathrm{CeFeSi}$ ).

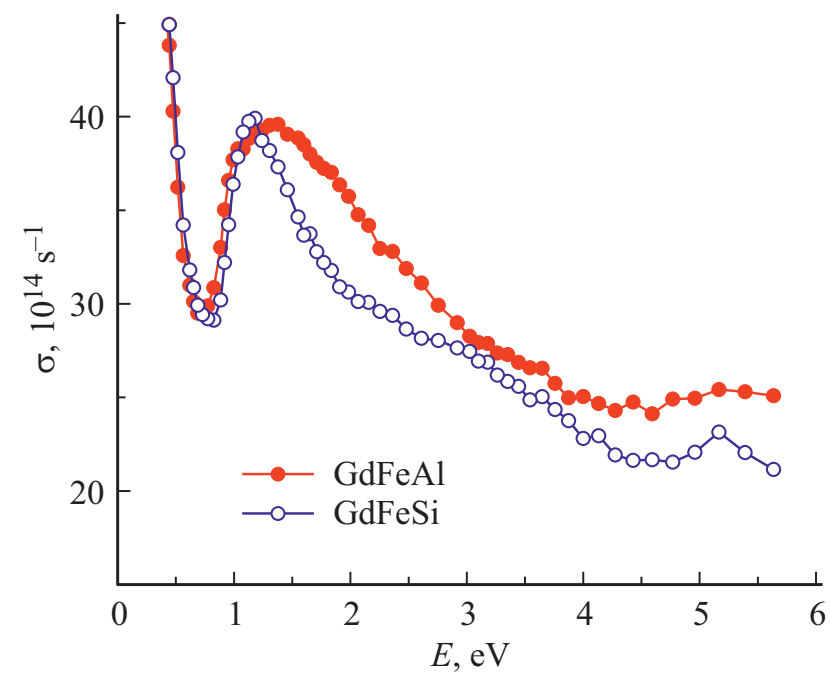

Рис. 4. Дисперсионные зависимости оптической проводимости соединений $\mathrm{GdFeAl}$ и $\mathrm{GdFeSi}$. 
Экспериментальные зависимости оптической проводимости $\sigma(\omega)$ для соединений $\mathrm{GdFeAl}$ и $\mathrm{GdFeSi}$ показаны на рис. 4. Представленные кривые имеют сходный профиль, характеризуемый резким спадом в низкоэнергетической области, сменяющимся крутым подъемом и последующим образованием максимумов при $1.2 \mathrm{eV}$ в $\mathrm{GdFeSi}$ и при $1.4 \mathrm{eV}$ в GdFeAl. При дальнейшем увеличении энергии фотона интенсивность данных структур быстро убывает. Характер этих зависимостей типичен для металлоподобных сред, когда в спектрах отчетливо выражены энергетические интервалы, соответствующие внутри- и межзонному поглощению света. В данном случае, при низких энергиях фотонов $E \lesssim 0.8 \mathrm{eV}$ (ИК-область), дисперсия кривых оптических проводимостей соответствует друдевскому типу $\sigma \sim \omega^{-2}$, описывающему взаимодействие свободных электронов с полем световой волны в пределах одной зоны. С ростом энергии фотонов (видимая и УФ-области) в спектрах $\sigma(\omega)$ наблюдаются изменения, связанные с проявлением механизма межзонного поглощения, что выражается в образовании интенсивных абсорбционных полос. Путем вычитания друдевской составляющей из экспериментальной зависимости $\sigma_{\text {inter }}(\omega)=\sigma(\omega)-\sigma_{\mathrm{D}}$ для каждого соединения можно выделить межзонный вклад в оптическую проводимость. Результат такой процедуры представлен на рис. 5. Существенно, что в спектрах $\sigma(\omega)$ обоих соединений в довольно широком интервале примерно до $\sim 3 \mathrm{eV}$ внутри- и межзонные вклады сосуществуют. Обращает внимание, что при выделении межзонных вкладов в зависимостях $\sigma_{\text {inter }}(\omega)$ в низкоэнергетической области появляется максимумы вблизи $0.4 \mathrm{eV}$, незаметные на экспериментальных кривых $\sigma(\omega)$ на фоне друдевского подъема. Как правило, спектр оптической проводимости в области квантового поглощения света для каждого материала содержит характерные особенности и определяется его электронной структурой. Хорошо видно, что форма полос поглощения в изучаемых соединениях также индивидуальна: в частности, в $\mathrm{GdFeAl}$ основная структура является более протяженной чем в $\mathrm{GdFeSi}$, а ее максимум локализован при более высоких энергиях. Кроме того, заметно различие в величинах интенсивности низкоэнергетического пика.

Природу формирования основных структур в спектрах $\sigma_{\text {inter }}(\omega)$ исследуемых соединений можно объяснить исходя из рассчитанных плотностей электронных состояний (рис. 1-3) с учетом того, что общая картина межзонного оптического поглощения в ферромагнитных материалах представляет собой суперпозицию вкладов от каждой из двух спин-поляризованных зон. Вычисление межзонных оптических проводимостей, соответствующих $\uparrow-$ и $\downarrow$-ориентациям спиновых моментов, было выполнено в соответствии с методом [30] на основе сверток плотностей электронных состояний, лежащих по обе стороны уровня Ферми. Суммарная проводимость $\sigma_{\text {inter }}(\omega)=\sigma \uparrow(\omega)+\sigma \downarrow(\omega)$, а также ее составляющие представлены на рис. 5. С учетом того, что в соединении $\mathrm{GdFeAl}$ реализовано двухфазное кристаллическое состо-
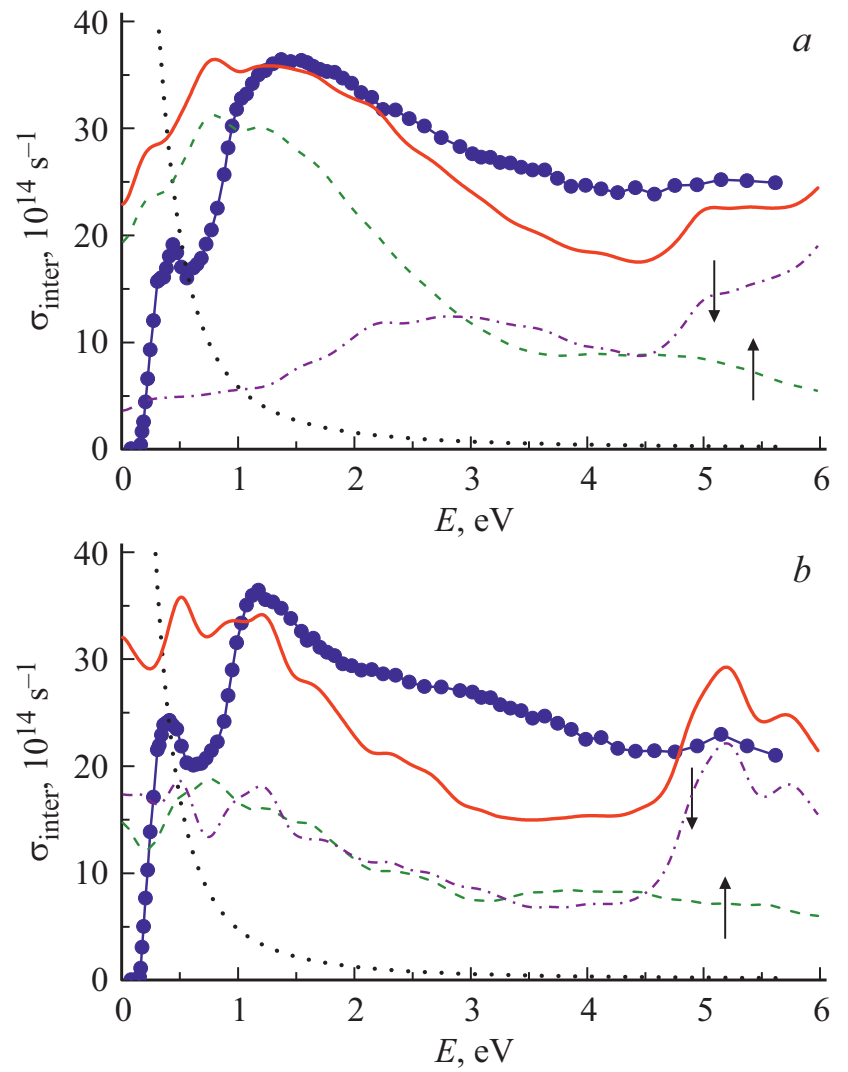

Рис. 5. Спектры межзонной оптической проводимости соединений $\mathrm{GdFeAl}(a)$ и $\mathrm{GdFeSi}(b)$. Точки - эксперимент, сплошная линия - расчет из полных плотностей состояний. Пунктир - друдевский вклад. Штриховые и штрих-пунктирные линии - парциальные вклады от электронных переходов в двух спиновых подсистемах.

яние, процедура расчета была двухступенчатой. Сначала спектры $\sigma_{\text {inter }}(\omega)$ рассчитывались для каждой из двух фаз отдельно, а затем производилось их усреднение с соответствующими весовыми множителями. Подчеркнем качественный характер вычислений, выполненных в приближении равенства вероятностей прямых и непрямых электронных переходов. Как следует из рисунка, в целом наблюдается заметное соответствие экспериментальных и теоретических кривых. На вычисленных зависимостях $\sigma_{\text {inter }}(\omega)$ выше $\sim 1 \mathrm{eV}$ отчетливо проявились основные особенности эмпирических спектров: присутствие максимумов и наличие почти монотонного спада на высокоэнергетическом склоне, переходящего при энергиях, близких к $5 \mathrm{eV}$, в заметный подъем. В то же время при $E \lesssim 1 \mathrm{eV}$ обращает внимание существенно различный характер поведения обсуждаемых зависимостей. В этой энергетической области ход рассчитанных кривых $\sigma_{\text {inter }}(\omega)$, в отличие от экспериментальных, указывает на то, что даже при $\omega \rightarrow 0$ вклад межзонного поглощения в оптическую проводимость остается большим. Такие аномально высокие значения низкоэнергетического поглощения, по нашему мнению, связаны 
с аппроксимациями, сделанными при расчете. К таким завышенным значениям низкочастотной проводимости, в первую очередь, приводит использование приближения постоянства матричных элементов электронных переходов, при котором переходы в пределах одной зоны имеют ненулевую вероятность.

На рис. 5 в рамках рассчитанной картины энергетических зон для обоих ферромагнитных соединений также представлены вклады в межзонную оптическую проводимость, связанные с каждой из двух спин-поляризованных зон. В соединении GdFeAl при энергиях ниже $\sim 3 \mathrm{eV}$, вычисления предсказывают доминирование электронных возбуждений в зонах со спином „вверх“, где интенсивные переходы происходят преимущественно между гибридизированными Fe $3 d \uparrow$ - и Gd $5 d \downarrow$-состояниями, разделенными уровнем Ферми. При этом природа формирования основного максимума в $\sigma_{\text {inter }}(\omega)$ при $1.4 \mathrm{eV}$ обусловлена переходами между зонами с повышенной плотностью состояний, которым соответствуют интенсивные пики в $N(E)$ по обе стороны от $E_{\mathrm{F}}$ (рис. 1 и 2). В системе электронов со спином „Вниз“, согласно расчетам, интенсивность межзонного поглощения существенно слабее, а его центр тяжести смещен в высокоэнергетическую сторону. В данном случае преобладающий вклад в $\sigma_{\text {inter }}(\omega)$ связан с переходами из Fe $3 d \downarrow$-зоны, характеризуемой максимумом $N(E)$ при $-2 \mathrm{eV}$ ниже $E_{\mathrm{F}}$, в $\mathrm{Gd} 5 d \downarrow$-состояния выше $E_{\mathrm{F}}$. В соединении $\mathrm{GdFeSi}$, как показывает рис. $5, b$, в процессе межзонного поглощения принимают участие обе системы спин-поляризованных электронов, а их вклад почти равнозначен до энергии $4.5 \mathrm{eV}$. Для обоих соединений рассчитанные зависимости $\sigma_{\text {inter }}(\omega)$ показывают наличие вблизи $\sim 5 \mathrm{eV}$ заметных структур в виде максимумов, образование которых связывается с электронными переходами из смешанных Fe $3 d \downarrow-$ Gd $5 d \downarrow-$-зон в Gd $4 f \downarrow$-зону. На эмпирических кривых $\sigma_{\text {inter }}(\omega)$ эти структуры проявились в более сглаженном виде. В целом можно говорить о качественном сходстве экспериментальных и теоретических частотных зависимостей межзонных оптических проводимостей исследуемых соединений. Это свидетельствует том, что проведенные расчеты электронной структуры дают адекватное описание их спектральных свойств в области квантового поглощения света.

\section{4. Заключение}

В работе приведены результаты спин-поляризованных $\mathrm{DFT}+U$-расчетов энергетических зависимостей полных и парциальных плотностей электронных состояний тройных соединений GdFeAl и GdFeSi. На базе этих данных вычислены спектры оптической проводимости в области квантового поглощения света, идентифицирована природа электронных состояний, участвующих в формировании спектров поглощения Оптические исследования, проведенные эллипсометрическим методом в широком спектральном интервале, свидетельствуют о качественном соответствии эксперимента с расчетными результатами. Показано, что структурные особенности экспериментальных частотных зависимостей межзонной оптической проводимости, за исключением низкоэнергетического интервала, удовлетворительно воспроизводятся в рамках теоретических расчетов данных функций.

\section{Финансирование работы}

Результаты исследований, содержащих расчет электронной структуры, выполнены при поддержке гранта Российского научного фонда (проект № 18-72-10098). Экспериментальные данные, связанные с синтезом соединений и оптическими измерениями, получены в рамках государственного задания Минобрнауки России (темы „Электрон“, № ААА-А18-118020190098-5 и „Магнит“, № АAА-А18-118020290129-5).

\section{Конфликт интересов}

Авторы заявляют, что у них нет конфликта интересов.

\section{Список литературы}

[1] S. Gupta, K.G. Suresh. J. Alloys Compd. 618, 562 (2015).

[2] Z. Hu, S. Bao-Gen. Chin. Phys. B. 24, 12, 127504 (2015).

[3] L. Li, M. Yan. J. Alloys Compd. 823, 153810 (2020).

[4] A.M. Palasyuk, B.Ya. Kotur, E. Bauer, H. Michor, G. Hilscher. J. Alloys Compd. 367, 205 (2004).

[5] D. Chen, A. Takeuchi, A.Inoue. J. Alloys Compd. 440, 199 (2007).

[6] M. Oboz, E. Talik. J. Alloys Compd. 509, 5441 (2011).

[7] S.A. Nikitin, T.I. Ivanova, I.A. Tskhadadze, K.P. Skokov, I.V. Telegina. J. Alloys Compd. 280, 16 (1998).

[8] R. Welter, G. Venturini, B. Malaman. J. Alloys Compd. 189, 49 (1992).

[9] H. Feng, H. Guo, X. Tao, H. Chen, Y. Ouyang, Y. Du, Y. He. J. Phase Equilib. Diffus. 34, 116 (2013).

[10] V. Provenzano, A.J. Shapiro, R.D. Shull, T. King, E. Canavan, P. Shirron, M. DiPirro. J. Appl. Phys. 95, 11, 6909 (2004).

[11] M. Klimczak, E. Talik. J. Phys.: Conf. Ser. 200, 092009 (2010).

[12] Q.Y. Dong, B.G. Chen, J. Chen, J. Shen, H.W. Zhang, J.R. Sun. J. Appl. Phys. 105, 7, 07A305 (2009).

[13] J. Kaštil, P. Javorský, J, Kamarád, L.V.B. Diop, O. Isnard, Z. Arnold. Intermetallics. 54, 15 (2014).

[14] P. Wlodarczyk, L. Hawelek, P. Zackiewicz, T. Rebeda Roy, A. Chrobak, M. Kaminska, A. Kolano-Burian, J. Szade. Mater. Chem. Phys. 162, 273 (2015).

[15] M. Napoletano, F. Canepa, P. Manfrinetti, F. Merlo. J. Mater. Chem. 10, 7, 1663 (2000).

[16] H. Drulis, W. Petriński, B. Staliński. J. Less-Commun. Met. 101, 229 (1984).

[17] B. Chevalier, M. Duttine, A. Wattiaux. Z. Naturforsch. 71B, 5, 419 (2016).

[18] I.A. Ovchenkova, S.A. Nikitin, I.S. Tereshina, A.Yu. Karpenkov, Y.A. Ovchenkov, J. Ćwik, Yu.S. Koshkid'ko, H. Drulis. J. Appl. Phys. 128, 14, 143903 (2020).

[19] S.A. Nikitin, T.I. Ivanova, I.A. Tskhadadze. Acta Phys. Polonica A 91, 2, 463 (1997). 
[20] A.G. Kuchin, S.P. Platonov, V.S. Gaviko, M.Yu. Yakovleva. J. Phys.: Conf. Ser. 1389, 012128 (2019).

[21] J. Jarosz, E. Talik. J. Alloys Compd. 317-318, 385 (2001).

[22] S. Talakesh, Z. Nourbakhsh. J. Supercond. Nov. Magn. 30, 2143 (2017).

[23] X.B. Liu, Z. Altounian. J. Appl. Phys. 107, 9, 09E103 (2010).

[24] S. Talakesh, Z. Nourbakhsh. Indian J. Phys. 93, 5, 571 (2019).

[25] P. Giannozzi, O. Andreussi, T. Brumme, O. Bunau, M. Buongiorno Nardelli, M. Calandra, R. Car, C. Cavazzoni, D. Ceresoli, M. Cococcioni, N. Colonna, I. Carnimeo, A. Dal Corso, S. de Gironcoli, P. Delugas, R.A. DiStasio Jr., A. Ferretti, A. Floris, G. Fratesi, G. Fugallo, R. Gebauer, U. Gerstmann, F. Giustino, T. Gorni, J. Jia, M. Kawamura, H.-Y. Ko, A. Kokalj, E. Küçkbenli, M. Lazzeri, M. Marsili, N. Marzari, F. Mauri, N.L. Nguyen, H.-V. Nguyen,

A. Otero-de-la-Roza, L. Paulatto, S. Poncé, D. Rocca, R. Sabatini, B. Santra, M. Schlipf, A.P. Seitsonen, A. Smogunov, I. Timrov, T. Thonhauser, P. Umari, N. Vast, X. Wu, S. Baroni. J. Phys.: Condens. Matter 29, 46, 465901 (2017).

[26] V.I. Anisimov, F. Aryasetiawan, A.I. Lichtenstein. J. Phys.: Condens. Matter 9, 4, 767 (1997).

[27] J. P. Perdew, K. Burke, M. Ernzerhof. Phys. Rev. Lett. 77, 18, 3865 (1996).

[28] M. Topsakal, R.M. Wentzcovitch. Comput. Mater. Sci. 95, 263 (2014).

[29] H. Fujiwara. Spectroscopic Ellipsometry: Principles and Applications. John Wiley Sons., N. Y. (2007). 392 p.

[30] I.I. Mazin, D.J. Singh, C. Ambrosch-Draxl. Phys. Rev. B 59, 1, 411 (1999).

Редактор К.В. Емцев 